ApplicationForum

\title{
Reducing Tissue Autofluorescence
}

\section{Innovations in immunofluorescent analysis boost signal-to-noise ratios}

Timothy Karpishin, Ph.D. Director of Chemistry, Vector Laboratories, Inc. tim@vectorlabs.com

\section{Introduction}

Immunofluorescence is a common technique in modern biology. It is a highly sensitive method used for the analysis of tissue samples with a fluorescence microscope. In this technique, antibodies are labeled with a fluorescent molecule in order to localize the antigen-antibody complex. Immunofluorescence (IF) can provide clear subcellular visualization of proteins, glycans, and small molecules. However, tissue autofluorescence often prevents the effective use of this tool. As will be described, a new technology has recently been developed that significantly lowers autofluorescence and leads to dramatic enhancement in signal-to-noise ratios.

Autofluorescence is a general term describing the background fluorescence in tissue sections unrelated to the specific signal generated during an IF assay. Tissue components such as red blood cells (RBCs) and collagen are strongly fluorescent, making it difficult to discern between relevant signal and background. In addition, formalin fixation, which is commonly used to immobilize antigens while retaining cellular structure, introduces a significant amount of fluorescence. The presence of autofluorescence makes interpretation of assay results particularly troublesome with green- and red-channel fluorophores.

Historically, the main method that has been employed to lower autofluorescence has been to treat the tissue with solutions of Sudan Black or similar dyes. These hydrophobic dye molecules can bind to tissue sections and lower fluorescence through absorption of incident radiation. Specifically, Sudan Black is effective at lowering the fluorescence due to lipofuscin. Lipofuscin is a brightly fluorescent pigment that is composed of lipid-containing residues. Because of the hydrophobic nature of Sudan Black, it binds effectively to the lipofuscin granules and masks their fluorescence. However, Sudan Black is much less effective at lowering the fluorescence due to aldehyde fixation, connective tissue elements, and RBCs.

\section{New Technology}

A new method has now been introduced that allows for the dramatic reduction of the autofluorescence due to formalin fixation, collagen, elastin, and RBCs. This innovative technology, sold as the Vector ${ }^{\circledR}$ TrueVIEW $^{\mathrm{TM}}$ Autofluorescence Quenching Kit, involves the treatment of
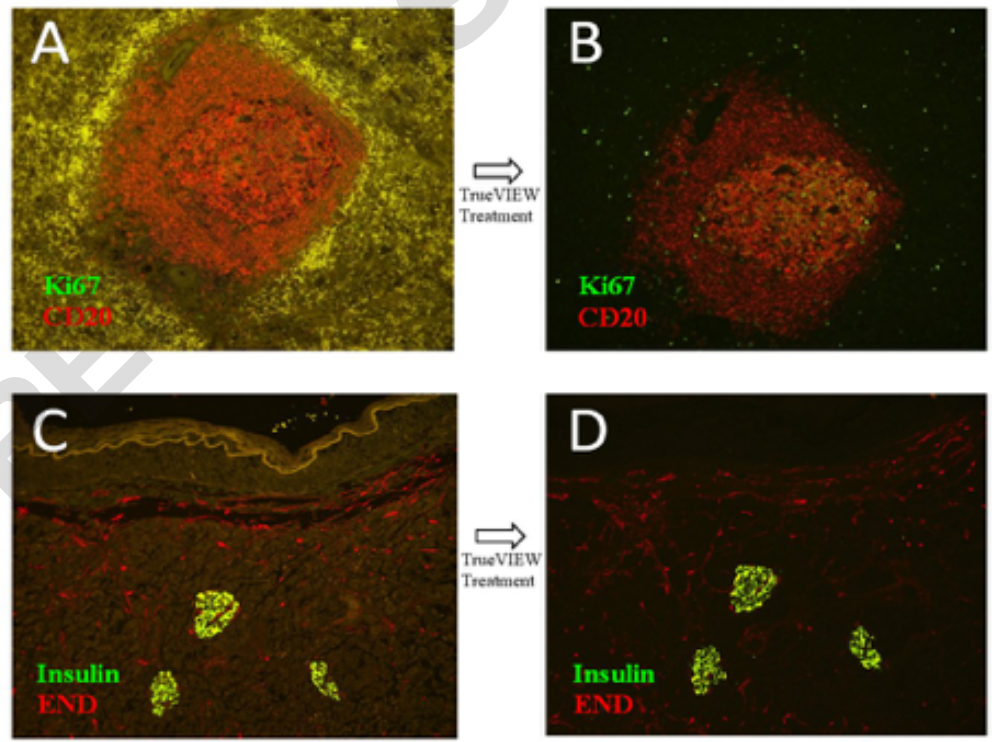

Figure 1. Dramatic reduction of autofluorescence reveals true localization of target antigen. FFPE human spleen probed with anti-Ki67 (green) and anti-CD20 (red), without (A) and with TrueVIEW ${ }^{T M}$ treatment (B). FFPE human pancreas probed with anti-insulin (green) and anti-END (red), without (C) and with TrueVIEW ${ }^{\mathrm{TM}}$ treatment (D). tissue with an aqueous solution of a hydrophilic molecule that binds electrostatically to collagen, elastin, and RBCs. This non-fluorescent molecule also binds effectively to formalin-fixed tissue including colon, pancreas, prostate, tonsil, spleen, kidney, gallbladder, and thymus. Once bound, the TrueVIEW TM Quencher significantly lowers the fluorescence of tissue components. Treatment with the TrueVIEW ${ }^{\mathrm{TM}}$ Quencher only requires a two-minute step and is compatible with all common fluorophores such as fluorescein, AlexaFluor ${ }^{\circledR}$ dyes, DyLight ${ }^{\circledR}$ fluors, cyanine fluors, and green fluorescent protein.

Figure 1 demonstrates the usefulness of the TrueVIEW'M Quencher in analysis of spleen and pancreas tissue. Prior to treatment, considerable autofluorescence is seen in the green channel that obfuscates the signal due to Ki67 (Figure 1, A). After treatment, a clear representation of Ki67 targets is seen as bright green punctate staining on a black background (Figure 1, B). Figure 1 also demonstrates the effect of using the TrueVIEW'M Quencher with pancreas tissue, where both green and red background autofluorescence is eliminated (Figure 1, C and D).

For the expanding field of immunofluorescence, a new method has now been developed that is rapid, easy to use, and allows the researcher to use formalin fixed specimens that were previously unacceptable due to autofluorescence. The Vector ${ }^{\circledR}$ TrueVIEW $^{T M}$ Autofluorescence Quenching Kit is broadly applicable for most tissue-based IF assays. 